\title{
A model for porcine reproductive and respiratory syndrome with time-dependent infection rate: traveling wave solution
}

\author{
Jeerawan Suksamran ${ }^{1,2}$, Yongwimon Lenbury ${ }^{1,2^{*}}$, Pairote Satiracoo ${ }^{1,2}$ and Chontita Rattanakul ${ }^{1,2}$
}

\author{
"Correspondence: \\ scylb@yahoo.com \\ ${ }^{1}$ Centre of Excellence in \\ Mathematics, CHE, 328 Si Ayutthaya \\ Road, Phayathai, Ratchathewi, \\ Bangkok, 10400, Thailand \\ 2Department of Mathematics, \\ Mahidol University, Rama VI Road, \\ Phayathai, Ratchathewi, Bangkok, \\ 10400, Thailand
}

\begin{abstract}
Porcine reproductive and respiratory syndrome (PRRS) gives rise to reproductive disorders in sows and problem with respiratory system in piglets and young pigs. This disease creates serious economic losses to major pork producing countries. The disease, which is characterized by high morbidity and significant mortality, combined with its potential for rapid spread, can devastate the pig industries of the affected countries. However, not much is known about the spatial transmission of PRRSV (porcine reproductive and respiratory syndrome virus) in growing pigs. In previous models, the infection rate has been assumed to be constant with time. Experimental studies on specific cases of this viral infection suggest that this assumption might not hold. A structured model for the spread of PRRSV has therefore been constructed, incorporating time and spatial dimensions as well as the decline of infection rate with time. Using the traveling wave coordinate and the modified extended hyperbolic tangent method, we derive analytical solutions to the model system. Stability and phase plane analyses are also carried out in order to gain insights into the spatial spread of PRRS as time progresses.
\end{abstract}

Keywords: PRRS; time-dependent infection rate; traveling waves; modified extended tanh method

\section{Introduction}

There are many epidemic diseases in the swine population such as swine fever disease, foot and mouth disease and Aujesky's disease. One of the most devastating diseases is porcine reproductive and respiratory syndrome (PRRS), which was first reported in the United States in 1987 [1]. Initially, the disease was named the "mystery swine disease". In 1990, the first European outbreak of the disease was reported in Germany [2], after which it spread throughout Europe [3].

In 1991, the epidemic of PRRS in the Netherlands was found in Dutch breeding farms [4] and then spread to most countries with swine industries. The causative agent, porcine reproductive and respiratory syndrome virus (PRRSV), was first isolated in Europe by Wensvoort et al. in 1991 [5] and then in the United States by Collins et al. [6].

High rates of morbidity from these infectious diseases in swine farms would cause such a severe financial loss to the country which is suffering from the epidemic as a whole that it is crucial that all efforts are brought to bear to discover as much as possible about

(c) The Author(s) 2017. This article is distributed under the terms of the Creative Commons Attribution 4.0 International License (http://creativecommons.org/licenses/by/4.0/), which permits unrestricted use, distribution, and reproduction in any medium, provided you give appropriate credit to the original author(s) and the source, provide a link to the Creative Commons license, and indicate if changes were made. 
these diseases, to predict their progress as well as discover optimal prevention and control strategies. In this endeavor, mathematical models of the diseases are needed to study the behavior of the disease and control strategies. Various research works on this subject cannot find out how to control the disease effectively. Therefore, a mathematical model of the disease is needed to study the behavior of the disease and possibility of control.

According to Charoensook et al. [7] the National Statistical Office (NSO) recorded the population of Thailand to be at about 67,070,000 inhabitants in 2009 and the gross domestic product (GDP) was US 3,939 per capita. The swine industry in Thailand has been expanding rapidly as one of the major livestock industries since the 1970s. Swine is one kind of farmed animals that has traditionally been an important part of the integrated farming system in Thailand. In particular, pork has become the second most important meat in Thai consumption, with average consumption in the late 1990s of about $4.7 \mathrm{~kg}$ per person per year (FAO Corporate Document Repository, 2002). Moreover, Thailand is an agricultural country with around $34 \%$ of the households throughout the country working in agriculture and $93 \%$ of them located in rural areas. The two major activities in the agriculture area are the cultivation of crops (54\%) and integrated crop-livestock farming (35\%) [7].

For decades, many researchers have experimented and investigated on PRRS in the laboratories. In 2001, Nodelijk et al. [4] proposed a quantitative approach to evaluate the effectiveness of vaccination under experimental conditions. They used two consecutive experimental designs to investigate whether PRRSV transmission among vaccinated pigs was reduced compared to control pigs and to estimate the reproduction parameter $R$.

In 2002, Mortensen et al. [8] attempted to quantify the likely routes of the transmission of PRRSV among a cohort of Danish sow herds. The design was a nested case-control study. A random sample of time-matched controls was used instead of all non-infection herds in the cohort.

In 2004, Van Gucht et al. [9] investigated the interaction between PRRSV and LPS (lipopolysaccharide) and described the effects of different pharmacological agents on the PRRSV-LPS induced disease. The central hypothesis throughout this study is that PRRSV-LPS induced respiratory disease results from an overproduction of pro inflammatory cytokines locally in the lungs.

In 2005, Wu et al. [10] showed that there is an association between PRRSV 2b protein, a minor structural component of the Porcine Reproductive and Respiratory Syndrome Virus, and intact virus particles, by locating the $2 \mathrm{~b}$ protein in the same sucrose density fraction as infectious virus by Western immunoblot assay. However, these experiments could not rule out the possibility that the presence of $2 \mathrm{~b}$ or another $10 \mathrm{kDa}$ protein in the structure of PRRSV in the same fraction was a result of independent co-migration with the virions.

In 2009, Pitkin et al. [11] developed a model of a swine production region and demonstrated the airborne spread of PRRS virus over a distance representative of building separation in commercial agriculture. They also quantified infectious virus in bio-aerosols and evaluated a method of biosecurity designed to reduce this risk. In addition, they identified meteorological risk factors associated with the presence of virus in bio-aerosols. The model provides crucial new information on the airborne spread of PRRS virus and offers a means to reduce this risk. 
In 2010, Evans et al. [12] proposed a model of a farrow-finish pig herd, investigated the within-herd transmission dynamics of PRRSV, and examined patterns of on-farm persistence and fade-out. The model was structured to represent the management of a typical European pig herd and run for various isolation practices of purchased gilts, contact structure, herd size and the frequency of re-introduction of infectious gilts.

In the same year, Wilson and Pantoja [13] presented mathematical models of host-PRRS virus interaction. The models are designed to explore the role of various components involved in the host response to PRRS virus infection identified by molecular studies, on the resulting infection characteristics. They presented a model of virus dynamics that can be further extended to include essential components of the cellular and humoral immune response.

Recently, there have been reports that infectiousness of pigs infected by the PRRSV is time dependent [14]. We therefore construct a structured model for the spread of PRRSV that incorporates both the time and the spatial dimensions as well as the decline of infectiousness with time. We derive analytical solutions to the model system by using the traveling wave coordinate, based on the modified extended tanh method [15], in order to gain insights into the spatial spread of the infection as time progresses.

\section{Model system}

Often, reaction-diffusion equations are used to describe the spread of populations in space. Hence, the other stream of research related to our work is on the reaction-diffusion equations.

In 1998, Chang and Manoranjan [16] studied a contaminant transport model with a cubic sorption isotherm and presented the method for finding exact solutions, which is a traveling wave front, by using traveling wave coordinate to obtain a coupled system of ordinary differential equations that can be reduced to a single second-order differential equation. Thus, they obtained exact solute concentration profiles for contaminant transport with the appropriate nonlinear sorption. Later, Manoranjan and Lee [17] studied Fishers equation, which is the logistic growth model which they modified to arrive at a population model that incorporates the efficiency of resource utilization based on the idea of adsorption theory in chemical kinetics. Traveling wave solutions were derived, possessing a constant wave speed $\geq 2$ in [17].

In 2011, Prasertsang et al. [18] proposed a dental plaque model consisting of a system of advective reaction-diffusion equations coupled with nonlinear sorption to describe the diffusion of mobile reactants through the tooth enamel and the time variation of the immobile reactants. Making use of the traveling wave coordinate, they transformed the partial differential equations into two coupled nonlinear ordinary differential equations and obtained the concentration profiles of the mobile reactant exactly in the form of a traveling wave front. Other examples of the use of traveling wave coordinates to find analytic solutions may be found in [19] and [20].

Based on the above works, we modify the reaction-diffusion equations which have been often used to describe the spread of infection to incorporate both time and spatial dimensions as well as the decline of infectiousness with time, arriving at the following model system:

$$
\frac{\partial S}{\partial t}=\frac{\partial^{2} S}{\partial x^{2}}+b_{S} S+b_{S I} I-\beta S \int_{0}^{t} \alpha e^{-\gamma(t-\tau)} I(x, \tau) d \tau-d_{S} S,
$$




$$
\frac{\partial I}{\partial t}=b_{I} I+\beta S \int_{0}^{t} \alpha e^{-\gamma(t-\tau)} I(x, \tau) d \tau-d_{I} I
$$

where $S(x, t)$ is the number of the susceptible swine at time $t$ and distance $x$ per unit area, and $I(x, t)$ is the number of the infected swine at time $t$ and spatial distance $x$ per unit area. The first term on the right of (1) is the diffusion rate of $S(x, t)$ in space, the second term is the birth rate from susceptible swine, the third term is the rate of birth of susceptible swine from infected swine, and the last term is the death rate of susceptible swine due to natural means or culling. The first and last terms in (2) are the birth and death rates of infectious swine, respectively.

The integral expression in (1)-(2) represents the rate of infection of susceptible swine due to infective swine $I(x, t)$. On considering the PRRS data reported by Charpin et al. [14], we observe that the number of new infections per infected swine decreases exponentially as time passes (Figure $3 \mathrm{~b}$ in the work of Charpin et al. [14]). For this reason, the exponential term $e^{-\gamma(t-\tau)}$ is utilized to represent the rate at which susceptible swine at time $t$ is infected by a swine infected at time $\tau$ earlier. Thus, the bigger $t-\tau$ is, the smaller the infection rate. The exponential term is multiplied by $I(x, t) d \tau$ to obtain the total rate of infection at time $t$ due to all swine infected at time $\tau$ earlier during the period $d \tau$. To get the total specific rate of infection at the time $t$, we integrate from 0 to $t$, to arrive at the integral expression which we shall denote by $G$ :

$$
G=\int_{0}^{t} \alpha e^{-\gamma(t-\tau)} I(x, \tau) d \tau
$$

Substituting (3) in (1)-(2) and differentiating G, we arrive at the following reactiondiffusion system model:

$$
\begin{aligned}
& \frac{\partial S}{\partial t}=\frac{\partial^{2} S}{\partial x^{2}}+b_{S} S+b_{S I} I-\beta S G-d_{S} S, \\
& \frac{\partial I}{\partial t}=b_{I} I+\beta S G-d_{I} I, \\
& \frac{\partial G}{\partial t}=\alpha I-\gamma G .
\end{aligned}
$$

We then introduce the traveling wave coordinate $\xi=x-c t$, where $c$ is the constant speed at which the wave is assumed to be moving. By using $\xi$ in (4)-(6), we obtain the following system of nonlinear ordinary differential equations:

$$
\begin{aligned}
& -c U^{\prime}=U^{\prime \prime}+\left(b_{U}-d_{U}\right) U+b_{U V} V-\beta U W, \\
& -c V^{\prime}=\beta U W+\left(b_{V}-d_{V}\right) V, \\
& -c W^{\prime}=\alpha V-\gamma W
\end{aligned}
$$

where ()$^{\prime}$ denotes the derivative with respect to $\xi, U(\xi)=S(x, t), V(\xi)=I(x, t)$ and $W(\xi)=$ $G(x, t)$. By using (9), we obtain $V^{\prime}=\frac{-c W \prime \prime}{\alpha}+\frac{\gamma W \prime}{\alpha}$. Substituting $V$ and $V^{\prime}$ into (7)-(9) we are led to the following system of second-order differential equations in terms of $U$ and $W$ :

$$
\begin{aligned}
& U^{\prime \prime}=-c U^{\prime}-\left(b_{U}-d_{U}\right) U+\frac{c b_{U V} W^{\prime}}{\alpha}-\frac{\gamma b_{U V} W}{\alpha}+\beta U W, \\
& W^{\prime \prime}=\frac{\alpha \beta U W}{c^{2}}+\frac{\left(\gamma-b_{V}+d_{V}\right) W^{\prime}}{c}+\frac{\gamma\left(b_{V}-d_{V}\right) W}{c^{2}} .
\end{aligned}
$$


Letting $y_{1}=U, y_{2}=y_{1}^{\prime}, y_{3}=W, y_{4}=y_{3}^{\prime}$, we can write (10) and (11) as

$$
\begin{aligned}
& y_{1}^{\prime}=y_{2}, \\
& y_{2}^{\prime}=-\left(b_{U}-d_{U}\right) y_{1}-c y_{2}-\frac{\gamma b_{U V}}{\alpha} y_{3}+\frac{c b_{U V}}{\alpha} y_{4}+\beta y_{1} y_{3}, \\
& y_{3}^{\prime}=y_{4}, \\
& y_{4}^{\prime}=\frac{\gamma\left(b_{V}-d_{V}\right)}{c^{2}} y_{3}+\frac{\left(\gamma-b_{V}+d_{V}\right)}{c} y_{4}+\frac{\alpha \beta}{c^{2}} y_{1} y_{3} .
\end{aligned}
$$

Next, we shall analyze the system (12)-(15) for its stability.

\section{Model analysis}

Before we derive the exact solution, a dynamical analysis may be carried out on the model system written in the form of the system (12)-(15). The system possesses two equilibrium points, namely $E_{0}=(0,0,0,0)$ and $E_{1}=\left(-\frac{\gamma\left(b_{V}-d_{V}\right)}{\alpha \beta}, 0, \frac{\left(b_{U}-d_{U}\right)\left(b_{V}-d_{V}\right)}{\beta\left(b_{U V}+b_{V}-d_{V}\right)}, 0\right)$.

We note that the non-washout steady state $E_{1}$ exists only if

$$
b_{V}-d_{V}<0
$$

and

$$
\frac{b_{U}-d_{U}}{b_{U V}+b_{V}-d_{V}}<0
$$

in which case all components of $E_{1}$ will be positive.

The Jacobian matrix of the system (12)-(15) about an equilibrium point $\left(\overline{y_{1}}, \overline{y_{2}}, \overline{y_{3}}, \overline{y_{4}}\right)$ is

$$
\bar{J}=\left[\begin{array}{cccc}
0 & 1 & 0 & 0 \\
\beta \overline{y_{3}}-\left(b_{U}-d_{U}\right) & -c & \beta \overline{y_{1}}-\frac{\gamma b_{U V}}{\alpha} & \frac{c b_{U V}}{\alpha} \\
0 & 0 & 0 & 1 \\
\frac{\alpha \beta}{c^{2}} \overline{y_{3}} & 0 & \frac{\alpha \beta}{c^{2}} \overline{y_{1}}+\frac{\gamma\left(b_{V}-d_{V}\right)}{c^{2}} & -\frac{\left(b_{V}-d_{V}-\gamma\right)}{c}
\end{array}\right] .
$$

At the equilibrium point $E_{0}=(0,0,0,0)$, the Jacobian matrix becomes

$$
J_{0}=\left[\begin{array}{cccc}
0 & 1 & 0 & 0 \\
-\left(b_{U}-d_{U}\right) & -c & -\frac{\gamma b_{U V}}{\alpha} & \frac{c b_{U V}}{\alpha} \\
0 & 0 & 0 & 1 \\
0 & 0 & \frac{\gamma\left(b_{V}-d_{V}\right)}{c^{2}} & -\frac{\left(b_{V}-d_{V}-\gamma\right)}{c}
\end{array}\right]
$$

whose eigenvalues are

$$
\lambda_{1,2}=\frac{-c \pm \sqrt{c^{2}-4\left(b_{U}-d_{U}\right)}}{2}, \quad \lambda_{3}=\frac{\gamma}{c}, \quad \lambda_{4}=-\frac{\left(b_{V}-d_{V}\right)}{c} .
$$

Since $\lambda_{3}$ is positive, this equilibrium point is unstable. In fact, we can conclude that $E_{0}$ shall be a saddle point if $b_{U}-d_{U}<0$ or $b_{V}-d_{V}>0$.

Further, we state the following result concerning the non-washout steady state $E_{1}$. 
Figure 1 Phase portrait of the system (12)-(15) with $b_{S}-d_{S}=-1.017520494$,

$b_{l}-d_{l}=0.040524417, b_{S I}=0.2, \alpha=0.437838$,

$\beta=0.032432, c=0.402694, \mu=0.120808$ and

$\gamma=0.488649$.

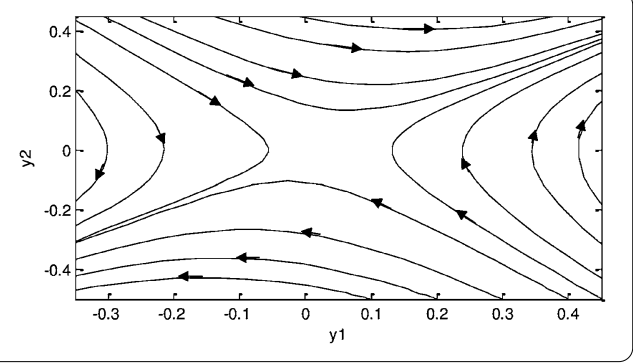

Theorem 3.1 The equilibrium solution $E_{1}$ of (12)-(15) is unstable for all positive parametric values whenever it exists.

Proof The Jacobian matrix of (12)-(15) about $E_{1}$ is

$$
J_{1}=\left[\begin{array}{cccc}
0 & 1 & 0 & 0 \\
\frac{-b_{U V}\left(b_{U}-d_{U}\right)}{b_{U V}+b_{V}-d_{V}} & -c & -\frac{\gamma\left(b_{U V}+b_{V}-d_{V}\right)}{\alpha} & \frac{c b_{U V}}{\alpha} \\
0 & 0 & 0 & 1 \\
\frac{\alpha\left(b_{U}-d_{U}\right)\left(b_{V}-d_{V}\right)}{c^{2}\left(b_{U V}+b_{V}-d_{V}\right)} & 0 & 0 & -\frac{\left(b_{V}-d_{V}-\gamma\right)}{c}
\end{array}\right]
$$

whose characteristic equation is

$$
\begin{aligned}
\lambda^{4} & +\left[\frac{\left(b_{V}-d_{V}-\gamma\right)}{c}+c\right] \lambda^{3}+\left[\left(b_{V}-d_{V}-\gamma\right)+\frac{b_{U V}\left(b_{U}-d_{U}\right)}{\left(b_{U V}+b_{V}-d_{V}\right)}\right] \lambda^{2} \\
& -\left[\frac{\gamma b_{U V}\left(b_{U}-d_{U}\right)}{c\left(b_{U V}+b_{V}-d_{V}\right)}\right] \lambda+\frac{\gamma\left(b_{U}-d_{U}\right)\left(b_{V}-d_{V}\right)}{c^{2}}=0 .
\end{aligned}
$$

According to the Routh-Hurwitz stability criterion, for the solution $E_{1}$ to be stable we need all coefficients in the characteristic equation above to be positive. However, the coefficient of $\lambda^{2}$ is

$$
\left(b_{V}-d_{V}-\gamma\right)+\frac{b_{U V}\left(b_{U}-d_{U}\right)}{\left(b_{U V}+b_{V}-d_{V}\right)}<0,
$$

since (16) and (17) must hold for this non-washout steady state $E_{1}$ to exist. Therefore, this equilibrium solution $E_{1}$ is unstable whenever it exists. This ends the proof of Theorem 3.1.

Figure 1 shows a phase portrait in the $y_{1}-y_{2}$ plane, in which the solution is seen to diverge from the origin, which is a saddle point as predicted.

Clinically, the above analysis implies that infection will not be controllable at a steady level, unless other measures are taken to remedy the situation.

\section{Analytical solution}

In this section, we shall rely on the modified extended tanh method [15, 21-23] to derive analytical solutions in terms of the traveling wave coordinate. First, the solution of the system (7)-(9) can be expressed as a finite series of tanh functions in the form

$$
U(\xi)=\sum_{m=0}^{M} a_{m} \phi^{m}
$$




$$
\begin{aligned}
& V(\xi)=\sum_{n=0}^{N} b_{n} \phi^{n}, \\
& W(\xi)=\sum_{h=0}^{H} c_{h} \phi^{h},
\end{aligned}
$$

where $a_{m}, b_{n}$ and $c_{h}$ are constants, and $\phi(\xi)=\tanh (\mu \xi)$ satisfying the Riccati equation

$$
\phi^{\prime}=\mu\left(1-\phi^{2}\right)
$$

We determine $M, N$ and $H$ by balancing the highest order of $\phi$ in the nonlinear term $U W$ with the highest order of $\phi$ in the linear term $U^{\prime \prime}$ in (7) which gives

$$
M+2=M+H,
$$

so that $H=2$. Balancing the highest order of $\phi$ in the term $U V$ with that in the term $V^{\prime}$ in equation (8) gives

$$
N+1=M+H=M+2,
$$

so that $N=M+1$. Balancing the term $W^{\prime}$ with the term $V$ in equation (9) gives

$$
H+2=N+3
$$

Therefore, we obtain

$$
M=2, \quad N=3 \quad \text { and } \quad H=2 .
$$

Substituting (21) into (18)-(20), we find that

$$
\begin{aligned}
& U(\xi)=a_{0}+a_{1} \phi+a_{2} \phi^{2}, \\
& V(\xi)=b_{0}+b_{1} \phi+b_{2} \phi^{2}+b_{3} \phi^{3}, \\
& W(\xi)=c_{0}+c_{1} \phi+c_{2} \phi^{2} .
\end{aligned}
$$

Substituting $\phi(\xi)=\tanh (\mu \xi)$ and the Riccati equation in equations (7)-(9), with the aid of (22)-(24), and equating the coefficients of each power of $\phi$, we obtain a system of algebraic equations of the parameters $a_{0}, a_{1}, a_{2}, b_{0}, b_{1}, b_{2}, b_{3}, c_{0}, c_{1}, c_{2}$, namely:

$$
\begin{aligned}
& 2 \mu^{2} a_{2}+b_{U} a_{0}-d_{U} a_{0}+b_{U V} b_{0}-\beta a_{0} c_{0}+c \mu a_{1}=0, \\
& 2 c \mu a_{2}-2 \mu^{2} a_{1}+b_{U} a_{1}-d_{U} a_{1}+b_{U V} b_{1}-\beta a_{0} c_{1}-\beta a_{1} c_{0}=0, \\
& -c \mu a_{1}-8 \mu^{2} a_{2}+b_{U} a_{2}-d_{U} a_{2}+b_{U V} b_{2}-\beta a_{0} c_{2}-\beta a_{1} c_{1}-\beta a_{2} c_{0}=0, \\
& -2 c \mu a_{2}+2 \mu^{2} a_{1}+b_{U V} b_{3}-\beta a_{1} c_{2}-\beta a_{2} c_{1}=0, \\
& 6 \mu^{2} a_{2}-\beta a_{2} c_{2}=0, \\
& b_{V} b_{0}-d_{V} b_{0}+\beta a_{0} c_{0}+c \mu b_{1}=0,
\end{aligned}
$$




$$
\begin{aligned}
& b_{V} b_{1}-d_{V} b_{1}+\beta a_{0} c_{1}+\beta a_{1} c_{0}+2 c \mu b_{2}=0, \\
& b_{V} b_{2}-d_{V} b_{2}+\beta a_{0} c_{2}+\beta a_{1} c_{1}+\beta a_{2} c_{0}-c \mu b_{1}+3 c \mu b_{2}=0, \\
& b_{V} b_{3}-d_{V} b_{3}+\beta a_{1} c_{2}+\beta a_{2} c_{1}+2 c \mu b_{2}=0, \\
& \beta a_{2} c_{2}-3 c \mu b_{3}=0 \\
& \alpha b_{0}-\gamma c_{0}+c \mu c_{1}=0 \\
& \alpha b_{1}-\gamma c_{1}+2 c \mu c_{2}=0 \\
& \alpha b_{2}-\gamma c_{2}-c \mu c_{1}=0, \\
& \alpha b_{3}-2 c \mu c_{2}=0 .
\end{aligned}
$$

Solving (25), we can obtain the parameters $a_{1}, a_{2}, b_{1}, b_{2}, b_{3}, c_{1}, c_{2}$ expressed in terms of $a_{0}, b_{0}, c_{0}$, which correspond to the initial boundary values of (4)-(6), as follows:

$$
\begin{aligned}
& a_{1}=\frac{\left(b_{U}-d_{U}\right) c a_{0}+c b_{U V} b_{0}-c \beta a_{0} c_{0}-\mu b_{U V} b_{1}+\mu \beta a_{0} c_{1}}{\mu\left(b_{U}-d_{U}-c^{2}-2 \mu^{2}-\beta c_{0}\right)}, \\
& a_{2}=\frac{b_{U V} b_{2}-\beta a_{0} c_{2}-\beta a_{1} c_{1}-c \mu a_{1}}{8 \mu^{2}-b_{U}+d_{U}+\beta c_{0}}, \\
& b_{1}=\frac{\left(d_{V}-b_{V}\right) b_{0}-\beta a_{0} c_{0}}{c \mu}, \quad b_{2}=\frac{\left(d_{V}-b_{V}\right) b_{1}-\beta\left(a_{0} c_{1}+a_{1} c_{0}\right)}{2 c \mu}, \\
& b_{3}=\frac{2\left(\left(b_{V}-d_{V}\right) b_{2}+\beta\left(a_{0} c_{2}+a_{1} c_{1}+a_{2} c_{0}\right)-c \mu b_{1}\right)-3 \beta\left(a_{1} c_{2}+a_{2} c_{1}\right)}{3\left(b_{V}-d_{V}\right)}, \\
& c_{1}=\frac{\gamma c_{0}-\alpha b_{0}}{c \mu}, \quad c_{2}=\frac{\gamma c_{1}-\alpha b_{1}}{2 c \mu},
\end{aligned}
$$

where

$$
\begin{array}{ll}
\alpha=\frac{2 b_{U V} a_{2} c_{2}^{2}}{a_{2}^{2}\left(3 b_{3} c_{1}+2 c_{2}\right)+a_{1} b_{3} c_{2}\left(3 a_{2}-1\right)}, & \beta=\frac{3 b_{U V} a_{2} b_{3}^{2}}{a_{2}^{2}\left(3 b_{3} c_{1}+2 c_{2}\right)+a_{1} b_{3} c_{2}\left(3 a_{2}-1\right)}, \\
c=\sqrt{\frac{2 b_{U V} a_{2}^{2} c_{2}}{2^{2}\left(3 b_{3} c_{1}+2 c_{2}\right)+a_{1} b_{3} c_{2}\left(3 a_{2}-1\right)}}, \quad \gamma=\frac{\left(2 b_{2} c_{2}-b_{3} c_{1}\right) b_{U V} a_{2}}{a_{2}^{2}\left(3 b_{3} c_{1}+2 c_{2}\right)+a_{1} b_{3} c_{2}\left(3 a_{2}-1\right)},
\end{array}
$$

and

$$
\mu=\frac{1}{2} \sqrt{\frac{2 b_{U V} b_{3}^{2} c_{2}}{a_{2}^{2}\left(3 b_{3} c_{1}+2 c_{2}\right)+a_{1} b_{3} c_{2}\left(3 a_{2}-1\right)}} .
$$

Thus, we obtain the exact solution of the model system:

$$
\begin{aligned}
S(x, t)= & a_{0}+\frac{\left(b_{S}-d_{S}\right) c a_{0}+c b_{S I} b_{0}-c \beta a_{0} c_{0}-\mu b_{S I} b_{1}+\mu \beta a_{0} c_{1}}{\mu\left(b_{S}-d_{S}-c^{2}-2 \mu^{2}-\beta c_{0}\right)} \tanh (\mu(x-c t)) \\
& +\frac{b_{S I} b_{2}-\beta a_{0} c_{2}-\beta a_{1} c_{1}-c \mu a_{1}}{8 \mu^{2}-b_{S}+d_{S}+\beta c_{0}} \tanh ^{2}(\mu(x-c t))
\end{aligned}
$$



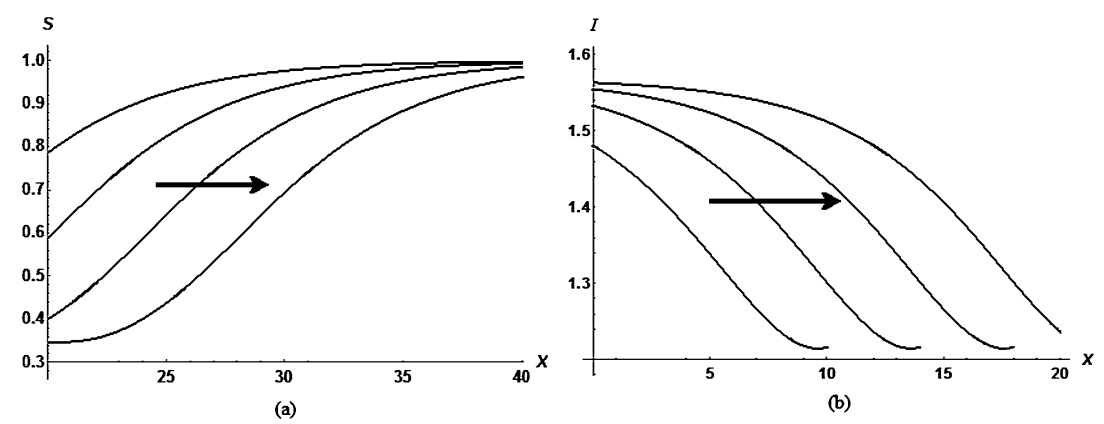

Figure 2 Travelling wave solution. (a) The susceptible population per unit area and (b) the infected population per unit area are plotted as functions of $x$ for $t$ between 30 (left most) and 60 (right most), plotted in time steps of 10 . Here, $S(0,0)=0.4, I(0,0)=1.285451998, G(0,0)=0.8186870039$, and other parametric values are as given in the text.

$$
\begin{aligned}
I(x, t)= & b_{0}+\frac{\left(d_{I}-b_{I}\right) b_{0}-\beta a_{0} c_{0}}{c \mu} \tanh (\mu(x-c t)) \\
& +\frac{\left(d_{I}-b_{I}\right) b_{1}-\beta\left(a_{0} c_{1}+a_{1} c_{0}\right)}{2 c \mu} \tanh ^{2}(\mu(x-c t)) \\
& +\frac{2\left(\left(b_{I}-d_{I}\right) b_{2}+\beta\left(a_{0} c_{2}+a_{1} c_{1}+a_{2} c_{0}\right)-c \mu b_{1}\right)-3 \beta\left(a_{1} c_{2}+a_{2} c_{1}\right)}{3\left(b_{I}-d_{I}\right)} \\
& \times \tanh ^{3}(\mu(x-c t)), \\
G(x, t)= & c_{0}+\frac{\gamma c_{0}-\alpha b_{0}}{c \mu} \tanh (\mu(x-c t))+\frac{\gamma c_{1}-\alpha b_{1}}{2 c \mu} \tanh ^{2}(\mu(x-c t)) .
\end{aligned}
$$

Figure 2 shows the graphs of the wave fronts $S(x, t), I(x, t)$ given in (26)-(27). Here, $a_{0}=$ $0.4, a_{1}=0.266667, a_{2}=0.333333, b_{0}=1.285451998, b_{1}=0.517284, b_{2}=1, b_{3}=0.2, c_{0}=$ $0.8186870039, c_{1}=0.7$, and $c_{2}=0.9$. The waves are seen here to travel from left to right as time increases.

The wave fronts seen in Figure 2 describe the situation where, at the center of the region of interest, $x=0$, the infection starts out being prevalent, while not that many healthy pigs remain (see Figure 2). As time passes, the number of infected cases here becomes higher, while the susceptibles reduce in number at this location. In other words, if we fix the location $x$, we observe the level of number of infectives to increase with time, but the number of susceptibles decreases.

On the other hand, if we fix the time, and follow a single wave front as $x$ increases, moving further and further away from the origin of infection, the susceptibles further away from the centre is higher in number per unit area because the infection needs time to spread outward before it reaches that distant location.

This can be more clearly understood by looking at Figure 3 which shows 3D plots of the levels of susceptible and infective populations as functions of $x$ and $t$. With this graph, we can find what would happen to the swine population at risk to this disease as we move in the direction of simultaneously increasing time and space.

\section{Conclusion}

In previous models [11-14], transmission since infection was assumed to be constant with time. However, Charpin et al. [14] have provided evidence that this is not the case. We 


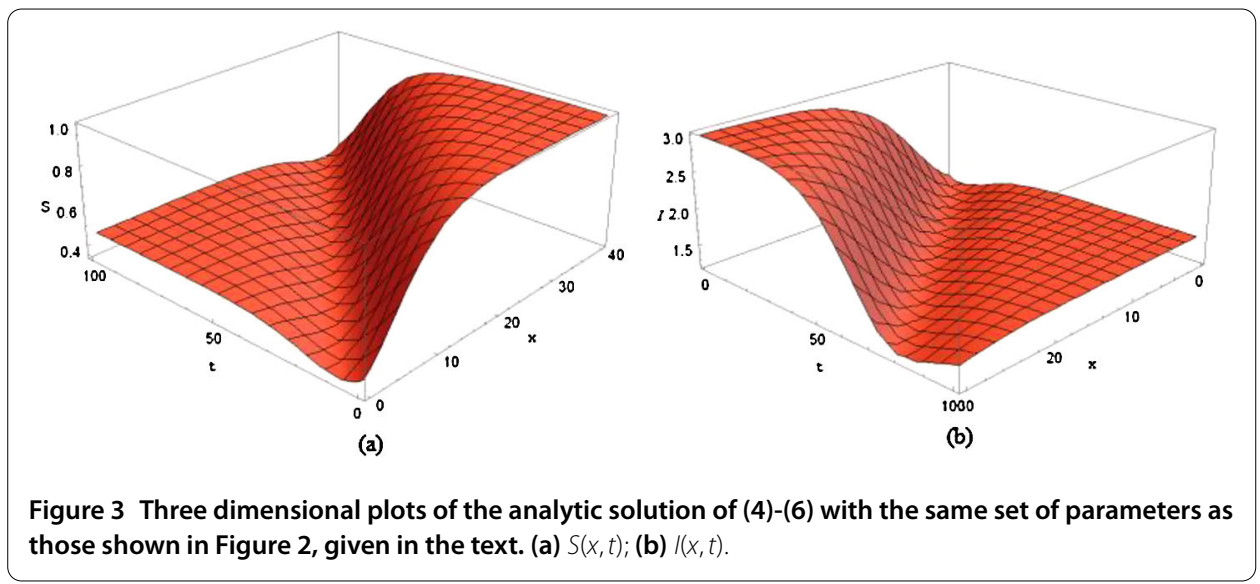

have therefore constructed a structured model for the spread of PRRSV that incorporates both the time and the spatial dimensions as well as the decline of infectiousness with time. We derive analytical solutions to the model system by using the traveling wave coordinate, based on the modified extended tanh method [16] and the earlier works on traveling wave solutions [17-22].

Our work is expected to form a basis for further investigation to test the potential effectiveness of employing various intervention strategies for disease containment, such as inoculation. Building upon our basic model, it should also be possible to examine other interventions aimed at reducing population contact rates as reinforcement to a containment policy.

Furthermore, in France, 50\% of French pigs are raised in areas like Brittany and hence the disease is prevalent in these densely pig populated regions. Some areas of France are, on the other hand, disease free [14]. Such heterogeneity in space should also be considered in a model of PRRS making the infection rate vary with space as well as time. Infection also appears to vary with the pigs' ages and this can be incorporated by building upon our basic model we have proposed here, which is a subject for future research.

According to Charpin et al. [14], porcine reproductive and respiratory syndrome leads to considerable financial losses. In addition, to deter bacterial secondary infections in pig production, farmers have to resort more and more to the use of antibiotics. In the United States, annual economic losses due to PRRS in its swine industry were estimated to be up to 664 million dollars in 2011. Therefore, it is important that knowledge on the occurrence and spread of PRRS virus in farms be deepened. The information obtained in this study could be useful to develop efficient strategies for PRRS surveillance not only in Thailand but in other countries as well. Further studies on PRRS incidence and persistence in farms are needed in order to prevent economic losses caused by the spread of this dangerous disease in the swine industry. 
Authors' contributions

$Y L$ introduced the research problem and modified the model to account for the drop in infectiousness observed in real data. JS analyzed the model, assisted by $Y L$, and derived the analytic solution. PS and CR provided advice on the stability analysis. All authors read and approved the final manuscript.

\section{Publisher's Note}

Springer Nature remains neutral with regard to jurisdictional claims in published maps and institutional affiliations.

Received: 18 January 2017 Accepted: 17 July 2017 Published online: 01 August 2017

\section{References}

1. Hill, H: Overview and history of mystery swine disease (swine infertility respiratory syndrome). In: Proc. Mystery Swine Disease Committee Meeting (1990)

2. Lindhaus, W, Lindhaus, B: Ratselhaft schweinekranheit. Prakt. Tierarzt 5, 423M25 (1991)

3. Terpstra, WJ: Serodiagnosis of bacterial diseases: problems and developments. Scand. J. Immunol. 36, 91-95 (1992)

4. Nodelijk, G, Jong, MCMD, Leengoed, LAMGV, Wensvoort, G, Pol, JMA, Steverink, PJGM, Verheijden, JHM: A quantitative assessment of the effectiveness of PRRSV vaccination in pigs under experimental conditions. Vaccine 19(27), 3636-3644 (2001)

5. Wensvoort, G, Terpstra, C, Pol, JMA, Laak, EAT, Bloemraad, M, Kluyver, EPD, Kragten, C, Buitten, LV, Besten, AD, Wagenaar, F, Broekhuhsen, JM, Moonen, PLJM, Zetstra, T, Boer, EAD, Tibben, HJ, Jong, MFD, Weld, PV, Groenland, GJR, Gennep, JAV, Voets, MT, Verheijden, JHM, Braamskamp, J: Mystery swine disease in the Netherlands: the isolation of Lelystad virus. Vet. Q. 13(3), 121-130 (1991)

6. Collins, JE, Benfield, DA, Christianson, WT, Harris, L, Hennings, JC, Shaw, DP, Goyal, SM, McCullough, S, Morrison, RB, Joo, HS, Gorcyca, D, Chladek, D: Isolation of swine infertility and respiratory syndrome virus (isolate ATCCVR-2332) in North America and experimental reproduction of the disease in gnotobiotic pigs. J. Vet. Diagn. Invest. 4(2), 117-126 (1992)

7. Charoensook, R, Knorr, C, Brenig, B, Gatphayak, K: Thai pigs and cattle production, genetic diversity of livestock and strategies for preserving animal genetic resources. Maejo Int. J. Sci. Technol. 7(01), 113-132 (2013)

8. Mortensen, S, Stryhn, H, Sogaard, R, Boklund, A, Stark, KDC, Christensen, J, Willeberg, P: Risk factor for infection of sow herds with porcine reproductive and respiratory syndrome (PRRS) virus. Prev. Vet. Med. 53(1-2), 83-101 (2002)

9. Gucht, SV, Labarque, G, Reeth, KV: The combination of PRRS virus and bacterial endotoxin as a model for multifactorial respiratory disease in pigs. Vet. Immunol. Immunopathol. 102(3), 165-178 (2004)

10. Wu, WH, Fang, Y, Rowland, RRR, Lawson, SR, Hennings, JC, Yoon, KJ, Nelson, EA: The 2b protein as a minor structural component of PRRSV. Virus Res. 114(1-2), 177-181 (2005)

11. Pitkin, A, Deen, J, Dee, S: Use of a production region model to assess the airborne spread of porcine reproductive and respiratory syndrome virus. Vet. Microbiol. 136(1-2), 1-7 (2009)

12. Evans, CM, Medley, GF, Creasey, SJ, Green, LE: A stochastic mathematical model of the within-herd transmission dynamics of porcine reproductive and respiratory syndrome virus (PRRSV): fade-out and persistence. Prev. Vet. Med. 93(4), 248-257 (2010)

13. Wilson, A, Pantoja, LG: Using mathematical models to gain insight into host-pathogen interaction in mammals: porcine reproductive and respiratory syndrome. In: The 2009 International PRRS Symposium, Chicago, Illinois (2009)

14. Charpin, C, Mahe, S, Keranflech, A, Belloc, C, Cariolet, R, Le Potier, MF, Rose, N: Infectiousness of pigs infected by the porcine reproductive and respiratory syndrome virus (PRRSV) is time-dependent. Vet. Res. 43(69), 1-8 (2012)

15. Taghizadeh, N, Mirzazadeh, M: The modified extended tanh method with the Riccati equation for solving nonlinear partial differential equations. Math. Æterna 2, 145-153 (2012)

16. Chang, CM, Manoranjan, VS: Travelling wave solutions of a contaminant transport model with nonlinear sorption. Math. Comput. Model. 28, 1-10 (1998)

17. Manoranjan, VS, Lee, I: Analysis of a population model with efficient resource utilization. J. Interdiscip. Math. 13, 41-50 (2010)

18. Prasertsang, P, Manoranjan, VS, Lenbury, Y: Analytical travelling wave solutions of a dental plaque model with nonlinear sorption. Nonlinear Stud. 18(1), 87-97 (2011)

19. Thiuthad, P, Manoranjan, VS, Lenbury, Y: Analytical solutions for an avian influenza epidemic model incorporating spatial spread as a diffusive process. East Asian J. Appl. Math. 5(2), 150-159 (2014)

20. Rattanakul, C, Lenbury, Y: Stability analysis and analytical solution of a nonlinear model for controlled drug release: trvelling wave front. Int. J. Math. Comput. Simul. 6, 351-359 (2012)

21. Hendi, $A$ : The extended tanh method and its applications for solving nonlinear nonlinear physical models. IJRRAS 3(1), 83-91 (2010)

22. Zedan, HA: New approach for tanh and extended-tanh methods with applications on Hirota-Satsuma equations Comput. Appl. Math. 28, 1-14 (2009)

23. Ibrahim, RS: Tanh-travelling wave solutions and reduction of variable-coefficient unstable nonlinear Schrodinger equation to a quadrature in electron bream plasma. Appl. Math. Sci. 2, 2157-2164 (2008) 